To appear in Engineering Optimization

Vol. 00, No. 00, Month 20XX, 1-22

\title{
Offshore Wind Farm Electrical Cable Layout Optimization
}

\author{
A.C. Pillai ${ }^{a b *}$, J. Chick $^{a}$, L. Johanning ${ }^{c}$, M. Khorasanchi ${ }^{d}$, and V. de Laleu ${ }^{b}$ \\ ${ }^{a}$ School of Engineering, The University of Edinburgh, Edinburgh, UK; \\ ${ }^{b}$ EDF Energy RED UK Centre, London, UK \\ ${ }^{c}$ College of Engineering, Mathematics, and Physical Sciences, University of Exeter, Penryn, UK \\ ${ }^{d}$ Faculty of Engineering, The University of Strathclyde, Glasgow, UK \\ (v6.0-R1 draft October 2014)
}

\begin{abstract}
This article explores an automated approach for the efficient placement of substations and the design of an inter-array electrical collection network for an offshore wind farm through the minimization of the cost. To accomplish this, the problem is represented as a number of sub-problems that are solved in series using a combination of heuristic algorithms. The overall problem is first solved by clustering the turbines to generate valid substation positions. From this, a navigational mesh pathifinding algorithm based on Delaunay triangulation is applied to identify valid cable paths, which are then used in a mixed-inter linear programming problem to solve for a constrained capacitated minimum spanning tree considering all realistic constraints. The final tree that is produced represents the solution to the inter-array cable results. This method is applied to a planned wind farm to illustrate the suitability of the approach and the resulting layout that is generated.
\end{abstract}

Keywords: Offshore wind farm layout optimization; inter-array cabling; clustering; pathfinding; capacitated minimum spanning tree

\section{Introduction}

Over the last decade the renewable energy sector has grown substantially and European governments are now targeting high levels of renewable energy penetration in the forthcoming decade. In order to achieve these ambitious targets, many utilities are looking to large offshore wind farms as part of the solution. Optimization of these large wind farms has therefore arisen as a growing field of research for both developers and academics.

The layout optimization problem arises primarily due to the variation of wind speed and therefore wind energy throughout a wind farm site. The variation is further intensified as all wind turbines operating in the wind produce a wake, a region of air directly behind the turbine where the wind speed is reduced and the turbulence intensity is increased. The effect of an upwind turbine's wake decreases the further downwind that a subsequent turbine is placed, however, the effect is still observed up to 20 rotor diameters downwind (Chamorro and Porté-Agel 2010). Further complicating matters, the cables that are needed to export the energy from each turbine have energy losses and costs which are associated with the length of cable and the cross-section of the cable. Also to be taken into consideration are the environmental and social constraints such as the seabed geology, local marine species, visual impact, shipping routes, and fishing areas to name a few. The layout optimization problem therefore becomes a problem of 
balancing the energy extraction from the wind; the system losses; the project costs; and the environmental and social constraints.

Many of the planned offshore wind farms in the UK, the Crown Estate Round 3 Projects, exceed $1 \mathrm{GW}$ in installed capacity and are expected to consist of several hundred individual wind turbines. In existing offshore wind farms, the turbines tend to be connected in strings of 5-10 turbines to a central collection point known as an offshore high voltage substation (OHVS). These substations are in turn connected to grid connection points onshore. As offshore sites offer little in regards to complex-terrain (i.e. hills, valleys, etc.) the turbines have until now generally been placed in straight lines along a regular grid. This, however, has not been optimized and early studies have indicated that optimization of the turbine positions can lead to more efficient use of the wind farm area (Fagerfjäll 2010; Elkinton 2007). Existing tools have approached the optimization of offshore wind farm layouts as a maximization of the energy yield and the minimization of wake losses, however, it can more accurately be characterized from a utility perspective as an optimization of the profitability of the generation asset or a minimization of the levelized cost of energy (LCOE). With regards to this, it therefore becomes important to consider all layout dependant aspects that either affect the energy yield of the wind farm or the lifetime costs.

The electrical infrastructure impacts both the energy yield and the costs and therefore has an important role to play in the optimization of offshore wind farms. The length of cable and therefore the capital costs of the project are directly a function of the positions of the turbines and the length of the cables also affects the energy losses that occur when transmitting through the cables. Similarly these lengths of cable depend on where the substations are placed relative to both the onshore connection point and the turbines. The optimization of the collection network, the cables, and substations, therefore forms an important component of the overall global optimization of an offshore wind farm layout.

In the development of a tool to be used to optimize the layouts of offshore wind farms, the problem of optimizing the electrical collection network for an offshore wind farm has been examined. Considering the future UK Round 3 projects as a point of context, the problem has been approached including as many realistic constraints as possible and formulated using a combination of heuristics and mixed-integer linear programming (MILP). As heuristics are used, this method may not reach proven optimality, but rather reaches a good feasible solution in an acceptable run time.

This optimization problem includes the determination of the substation positions given the realistic constraints faced by a developer, and the determination of the cable layout given this substation position. The export cable, a component of the transmission network, is not considered as part of this optimization problem.

Previous work in this field has tended to look at small wind farms, or has omitted some of the necessary constraints needed for the optimization of a real wind farm. Most have elected to work only on a single construction phase of a wind farm with a single OHVS, as subsequent phases and additional OHVS would follow the same procedure.

Fagerfjäll (2010) implemented an MILP based approach for the electrical cable layout, assuming that all the turbines were connected to a single substation. This approach used a variation on the minimum spanning tree problem, a minimum Steiner tree, in order to solve for the electrical cabling. A minimum Steiner tree is similar to a minimum spanning tree, however, the arcs may branch anywhere along an arc and not only at nodes. By approximating the problem to that of the minimum Steiner tree, the cable length is therefore further minimized. Similar work has also been undertaken by Svendsen (2013) and Lindahl et al. (2013) using a MILP implementation to solve for a capacitated minimum spanning tree. Both of these studies, however, correctly identified that the computational time for these problems grows very quickly with the number of turbines. In 
fact, the capacitated minimum spanning tree (CMST) problem is NP-hard and therefore an optimal solution is not found in polynomial time, but rather exponential. The problem therefore becomes exponentially complex as more turbines are added and more possible cable arcs must be considered.

Due to the complexity, a number of studies have opted to use heuristic algorithms such as genetic algorithms in order to optimize the electrical cable layout (Dutta and Overbye 2011; González-Longatt and Wall 2012; Cerveira and Pires 2014; Li, He, and Fu 2008; Zhao, Chen, and Blaabjerg 2008, 2009; Lumbreras and Ramos 2013). These studies have therefore sacrificed finding the proven optimal solution in favour of a good feasible solution in acceptable time-scales. Bauer and Lysgaard (2013) simplified the problem to only allowing strings of turbines without any branching, allowing a variation on a vehicle routing problem algorithm to be applied. This too finds solutions in reasonable timescales, however, by not allowing branching reduces the problem complexity significantly, and eliminates many feasible solutions unnecessarily including potentially the optimal solution.

Studies carried out by Dutta and Overbye (2011, 2012, 2013) have looked at using a minimum spanning tree (MST) and applying the capacity constraints by running the MST on clustered turbines representing the capacity constraints of the largest crosssection of cable. This work has also modified the MST to represent a minimum Steiner tree. Dutta and Overbye (2013) also include an algorithm to account for exclusion areas where cables may not be placed, by constructing convex hulls from the obstacle and turbine positions to derive a shortest path.

Given the desire to apply the methodology to real sites, the electrical inter-array cable optimization problem has been approached pragmatically, dividing the overall problem into two sub-problems: the placement of the substations and then the determination of the cable layout. The study at hand intentionally opted to continue on from the work of Fagerfjäll (2010); Svendsen (2013); Lindahl et al. (2013) using a MILP formulation for the electrical cable layout problem and introduce additional constraints to represent the realistic case of UK Round 3 sites. The new constraints introduced in this work take into account complex geographical information systems (GIS) shapes as constraints and the fact that cables may not cross in the offshore environment. Additional constraints have also been explored to aid in reducing the computational time.

\section{Process Overview}

The design of offshore wind farms and the decision regarding the number of substations to build is largely driven by the capital expenditure (CAPEX) associated with building a substation along with the necessary foundation works. Projects tend therefore to minimize the number of substations such that substations are efficiently designed with a minimum surplus capacity. The total number of substations is therefore often predetermined based on the number of construction phases or the total wind farm capacity.

As a result of this, the decision of where to place the substations is effectively a process of selecting the substation positions which will result in the minimum total collection network cable as this will minimize both costs and losses of the collection system. The export cable should also be considered, however, it has been previously shown that given the significant length of cable already required for the export cable when compared to the in-field cables and the high voltage levels used, the costs associated with the export cable are minimally impacted by changes in the substation positions (Fagerfjäll 2010). In order to address this problem it was therefore decided to break the problem into two sub-problems: first the determination of the substation positions and secondly the construction of a CMST representing the cabling for each substation and its assigned 
turbines.

In the offshore environment cable junctions require additional switch-gear and power electronics, the installation of which will require some sort of physical structure to house them. Presently all junction boxes and circuit breakers designed for the offshore wind sector are designed to be housed in a turbine or placed on a substation platform (Burton et al. 2011). This limitation in the offshore environment results in wind farm collection networks only branching at either turbines or substations. Though a minimum Steiner tree or a CMST with Steiner points would reduce the length of cable needed to connect a wind farm as proposed by Fagerfjäll (2010); Dutta and Overbye (2012, 2013), it is not feasible to implement a Steiner tree in the offshore environment. A CMST without Steiner points was therefore selected for use in this study as this better represents the physical constraints of offshore wind farms.

The CMST formulation requires costs for each potential cable connection under consideration. In order to assess this, it was first necessary to determine the length of cable required to connect two turbines, and then apply a per metre cost for that cable type. As the costs of cables including the installation costs scale with cable length it is necessary to determine the lengths of potential cables prior to running the CMST. This effectively introduces another sub-problem. Given the complex GIS constraints, this was addressed through the implementation of a pathfinding algorithm in order to ensure that the cables would not pass through the constrained regions. Additional constraints were also introduced in order to reflect that cables may not cross one another. The overall programme approach is outlined below:

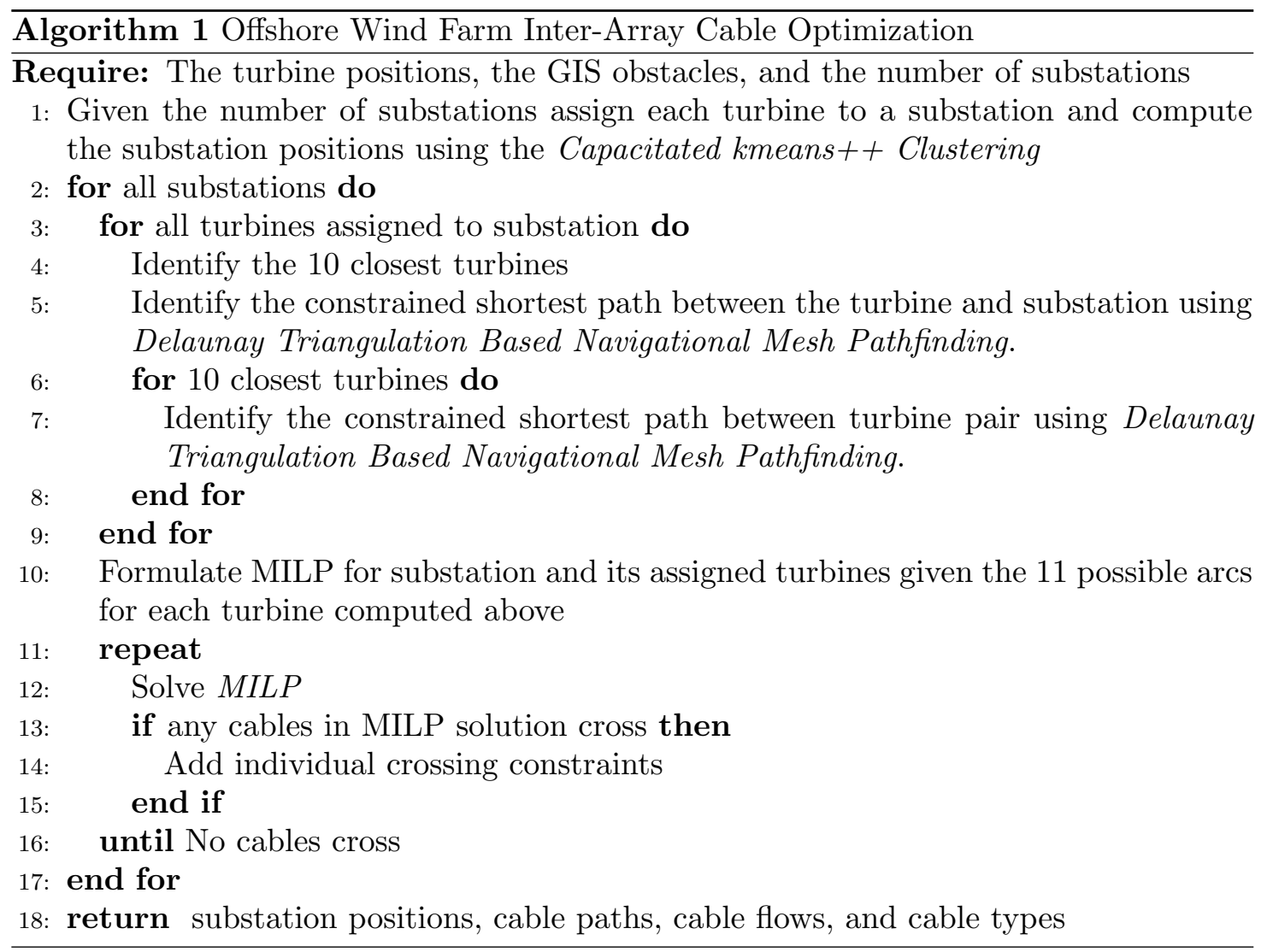

As shown in Algorithm 1, there are in fact three optimization sub-problems as part of this overall optimization:

(1) Capacitated Clustering Problem/Facility Location 
(2) Constrained Shortest Path/Pathfinding

(3) Construction of Constrained Capacitated Minimum Spanning Tree

The Constrained Shortest Path problem is executed for each turbine finding the possible connections between it, the ten closest turbines to it, and the substation. This data is used for the MILP CMST problem which is executed for each of the substations. The number of turbines to pathfind to is a parameter, and 10 was empirically selected as turbines were found to always be connected either to one of their six closest neighbours or the substation in all tests conducted. Ten was therefore selected to give additional flexibility, however, the framework is designed to accept any valid integer for this parameter.

Table 1. Notation for automated electrical network design

\begin{tabular}{lll}
\hline Name & Description & Type \\
\hline$A$ & All traversable points & Set \\
$L$ & All cable types & Set \\
$N_{t}$ & All turbines that can be connected to turbine $t$ & Set \\
$S$ & All substations & Set \\
$T$ & All turbines and substations & Set \\
$V$ & All turbine and substation positions, all vertices of the & Set \\
& full graph, $V=T \cup S$ & \\
$X_{l}$ & All cables that intersect cable arc $l$ & Set \\
$u_{i, j}$ & Arc between vertex $i$ and vertex $j$ is active in shortest & Binary Variable \\
& path & \\
$y_{i, j, l}$ & Presence of cable of type $l$ between nodes $i$ and $j$ & Binary Variable \\
$z_{t, s}$ & Assign turbine $t$ to substation $s$ & Binary Variable \\
$d_{i, j}$ & The arc length between vertex $i$ and vertex $j$ & Variable \\
$f_{i, j}$ & Flow between nodes $i$ and $j$ & Variable \\
$n_{s}$ & The number of turbines assigned to substation $s$ & Variable \\
$p_{1}$ & Source point & Variable \\
$p_{2}$ & Termination point & Variable \\
$x_{s}$ & The position in $x-y$ space of substation $s$ & Variable \\
$x_{t}$ & The position in $x-y$ space of turbine $t$ & Variable \\
$A_{l}$ & Cross-sectional area of cable type $l$ & Parameter \\
$c_{f}$ & Price of electricity & Parameter \\
$c_{l}$ & Cost of cable type $l$ per metre installed & Parameter \\
$g_{j}$ & Power generated at node $j$ & Parameter \\
$I$ & Current level at peak & Parameter \\
$Q_{c o n n e c t i o n}$ & Number of cables that can be connected to a turbine & Parameter \\
$Q_{l}$ & node & Power flow capacity of cable type $l$ \\
$Q_{s}$ & The capacity of substation $s$ & Parameter \\
$R$ & Cable resistivity & Parameter \\
\hline & & Parameter \\
\hline
\end{tabular}




\section{Substation Placement Based on $k$-means ++ Clustering}

\subsection{Problem Description}

The substation placement problem can be described that for $n_{t}$ turbines, $k$ substations must be placed optimally. As the overall problem seeks to design the inter-array cable paths the logical approach is to try and reduce these path lengths from the outset by efficiently placing the substations. The substation placement problem has therefore been addressed as a capacitated centred clustering problem (CCCP) and facility location problem. Based on the turbine positions and the number of substations desired, the turbines are divided into clusters each within the capacity of the substations.

\subsection{Problem Formulation}

Mathematically, the problem can be expressed as:

$$
\begin{array}{rlr}
\operatorname{minimize} & \sum_{t \in T} \sum_{s \in S}\left(x_{t}-x_{s}\right)^{2} z_{t, s} & \\
\text { subject to } & \sum_{s \in S} z_{t, s}=1 & \forall t \in T, \\
& \sum_{t \in T} z_{t, s}=n_{s} & \forall s \in S, \\
& \sum_{t \in T} x_{t} z_{t, s}=n_{s} x_{s} & \forall s \in S, \\
& \sum_{t \in T} z_{t, s} \leq Q_{s} & \\
& z_{t, s} \in\{0,1\} & \\
& x_{t} \in \mathrm{R}^{n} \quad x_{s} \in \mathrm{R}^{n} \quad n_{s} \in \mathrm{N} \quad \forall t \in T, \forall s \in S &
\end{array}
$$

where $T$ is the set of turbines and $S$ is the set of substations.

In the above formulation, equation 1a states the objective function of the optimization process which is to minimize the square of the Euclidean distance between the position $x_{s}$ of each substation, $s$, and the individual turbine positions $x_{t}$ if the turbine $t$ is assigned to substation $s$ denoted by the state of $z_{t, s}$. The variable $z_{t, s}$ is defined as 1 if the turbine $t$ is assigned to substation $s$, it is 0 otherwise. Equation $1 \mathrm{~b}$ limits each turbine to being connected to exactly one substation. Equation 1c defines the number of turbines assigned to substation $s$ to be given by $n_{s}$. Equation $1 \mathrm{~d}$ defines the geometric centroid of the turbines assigned to substation $s$ to be the position of the substation, and equation $1 \mathrm{e}$ ensures that each substation satisfies the capacity constraints $Q_{s}$.

\subsection{Solution Approach}

The CCCP as formulated above, is NP-complete and has previously been studied by Negreiros and Palhano (2006); Geetha, Poonthalir, and Vanathi (2009); Chaves and Lorena (2010). These studies have identified heuristic algorithms as well suited for solving this problem. Based on the comparative study by Negreiros and Palhano (2006) which compared heuristic approaches for the CCCP, it was decided to build a two-phase heuristic 
for this problem. The first stage would identify the ideal cluster centres ignoring the capacity and obstacle constraints, and the second phase would apply first the capacity constraints finding a good solution starting from the solution of the first stage, and finally once the capacity constraints were satisfied, the obstacle constraints would be applied to refine the solution. It is recognized that the implementation of a heuristic algorithm cannot ensure an optimal solution, and the substation positions generated by this algorithm represent only a feasible solution.

For the first phase, a kmeans ++ algorithm was selected. This is a variation on the wellknown kmeans clustering methodology which intelligently selects the initial cluster centre positions in order to improve performance (Arthur and Vassilvitskii 2006; MacQueen 1967). Both kmeans and kmeans ++ work by iteratively computing the cluster centre (geometric median) based on what turbines are assigned to the cluster, then based on the new geometric median, the turbines are each reassigned to the closest cluster centre. This process is repeated until the cluster centres converge. In general, both kmeans and $k m e a n s++$ have been shown to be effective clustering techniques (Negreiros and Palhano 2006).

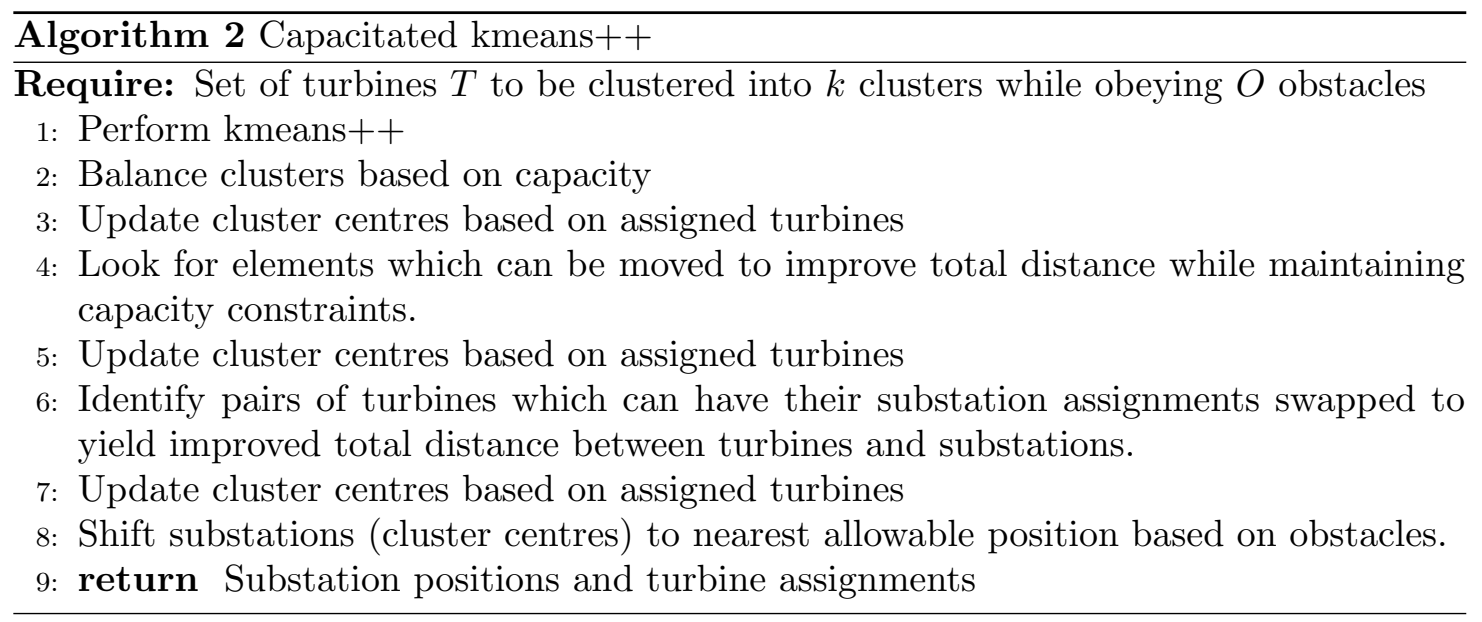

Using the approach outlined in algorithm 2 , it was possible to successfully partition a wind farm to ensure that substations were in good, feasible positions if not in the optimal position. This process also ensured that the substation capacities and any GIS obstacles were correctly implemented as constraints for the substation positions.

The proposed method also explored swapping turbine assignments in order to ensure that the identified substation positions accurately minimize the distance to turbines, and each turbine is therefore assigned to the closest substation unless capacity constraints are active in which case the turbines with the lowest global impact to the cost are assigned to a substation farther away. It should be noted that the result of introducing the GIS and capacity constraints has a major impact on the computational time of the clustering, but a very minor effect on the value of the objective function.

\section{Cable Path Creation Based on Delaunay Triangulation and Pathfinding}

\subsection{Problem Description}

Before constructing the capacitated minimum spanning tree it is necessary to compute the costs of putting a cable between two turbine locations. In order to do this while considering the GIS obstacle constraints, it was necessary to compute a constrained shortest path between the positions. Given the constraints, the construction of the graph 
of possible cable paths is an NP-Complete problem. Dutta and Overbye (2013) addressed exclusion areas by defining a bypassing algorithm. This bypassing algorithm constructs a convex hull of the obstruction and the turbines to be connected. The edge of this convex hull can then be traversed to find the shortest path. This approach, however, is not guaranteed to find the shortest path, and in fact will incorrectly mark areas as impassable if the obstacle is not convex. This bypassing algorithm is therefore only well suited if the exclusion areas can be described as simple convex shapes. As the tool developed here sought to account for realistic seabed constraints that may take on concave shapes it was decided that a convex hull based bypassing algorithm would not be the most efficient approach. As a result, a pathfinding approach was taken. The pathfinding approach was found to correctly account for concave obstacle regions.

Pathfinding can theoretically, depending on the algorithm applied, guarantee a shortest path between two points in a constrained configurational space regardless of if the obstacles are convex or not. Pathfinding problems frequently arise in video games and robot motion problems as it is necessary for a robot to move from an origin location to a destination location taking into account obstacles which it cannot pass through. In the case of cable paths, turbines are either connected by a cable to another turbine or the substation and therefore there is a finite set of origin-destination pairs for which a path must be found.

\subsection{Problem Formulation}

In general, pathfinding can be described as a specific case of a shortest path tree traversal. The shortest path of a graph can be mathematically formulated as:

$$
\operatorname{minimize} \sum_{i \in A} \sum_{j \in A} d_{i, j} \cdot u_{i, j}
$$

subject to $\sum_{i:(i, k) \in V} u_{i, k}-\sum_{j:(k, j) \in A} u_{k, j}= \begin{cases}-1, & \text { if } k=p_{1} \\ 1, & \text { if } k=p_{2} \\ 0, & \text { if }\left(k \in A: k \notin\left\{p_{1}, p_{2}\right\}\right)\end{cases}$

$$
u_{i, j} \in 0,1
$$$$
\forall(i, j) \in A
$$

where $u_{i, j}$ is a binary variable describing the connectivity between points $i$ and $j$ in space $A$ in the shortest path. This variable is 1 if $i$ and $j$ are connected in the shortest path and 0 otherwise. The points $p_{1}$ and $p_{2}$ represent the source and termination points respectively and are also with the space $A$. The cost of connecting points $i$ and $j$ (the length of the edge connecting $i$ and $j$ ) is given by $d_{i, j}$.

This general formulation, however, represents the optimization problem once a graph representing the configurational space, the traversable space in which cables can be laid, has been constructed. There are a number of different methods to construct this graph depending on what kind of pathfinding algorithm is deployed. For this study both a grid based pathfinding algorithm and a navigational mesh were implemented. The navigational mesh ultimately proved to be the more appropriate algorithm to implement.

\subsection{Solution Approach}

For problems such as this, there are two principle approaches for finding the shortest path, one is to reduce the obstacle data to a walkability grid representing on a regular grid where cables can and cannot be placed. The shortest path can then be found using 

grid.

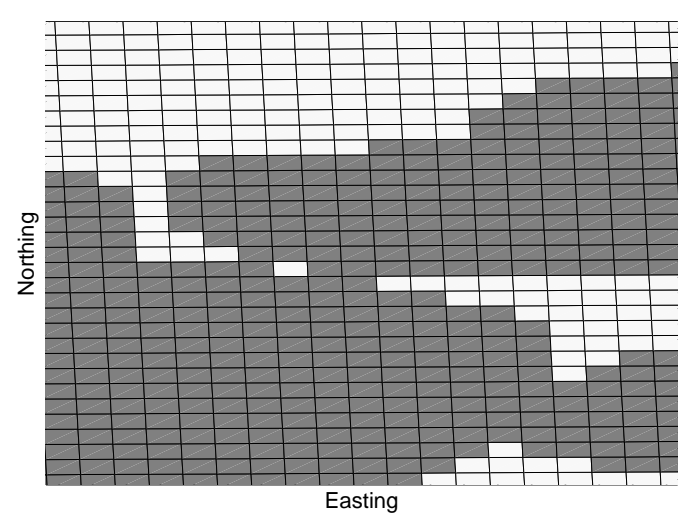

(a) Grid Based Pathfinding Obstacles

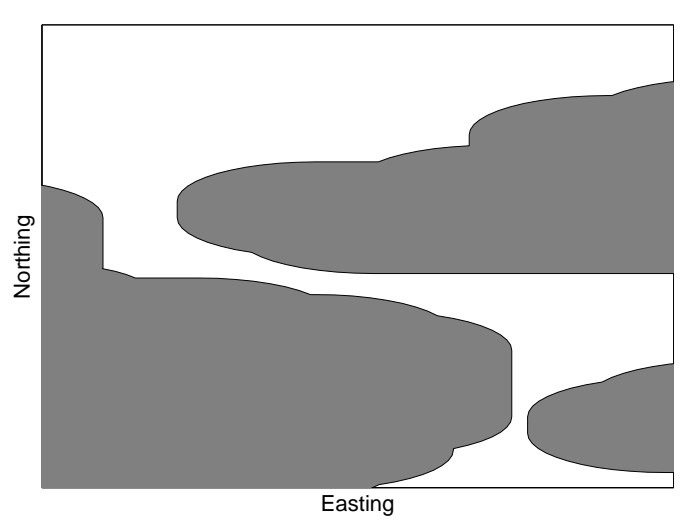

(b) Navigational Mesh Pathfinding Obstacles

Figure 1. Comparison of obstacle representation in grid based and navigational mesh based pathfinding.

a standard grid search algorithm such as $A^{*}$ Pathfinding or Dijkstra's. However, this simplifies all the constraints to consisting of regular rectangles and given the complexity of real offshore wind sites this was found to often eliminate possible paths as can be observed in figure 1. Though this could be avoided by using a finer grid size, other challenges still remained. For example, by creating a grid, the cable paths were limited in having only 8 options of where to go from any given grid position (fig. 2), often causing problems with paths overlapping cables near substations and no simple means of avoiding his. Paths based on the grid were also longer than necessary due to being fixed to the

Figure 1. Comparison of obstacle representation in grid based and navigational mesh based pathfinding.

The alternative method uses what is known as a visibility graph and navigational mesh, and is capable of avoiding all of the above problems, but at a significant cost in complexity (Ghosh 2007). The visibility graph is a graph for which an arc exists between any two vertices if they are 'visible' to one another. Visibility is defined as true if the two points can be connected by an arc without the arc passing through an obstacle. It is important to note that in terms of a visibility graph, points along the obstacle edges are considered to be an open set, that is that valid arcs can pass along edges. The optimal path is in fact the shortest path between vertices on such a graph. The difficulty in working with visibility graphs is that algorithms for testing visibility are computationally complex. The most efficient algorithms still operate in $O(n \log n+k)$ where $n$ is the number of vertices and $k$ is the number of edges (de Berg et al. 2008). Given that the GIS constraints for a typical offshore wind farm will constitute several thousand vertices this was thought to be too computationally complex.

The proposed methodology, therefore uses a heuristic algorithm which can create a close approximation of the visibility graph in a fraction of the computational time. This approach, known as a navigational mesh based pathfinding algorithm creates a traversable graph which obeys the obstacle constraints. One such algorithm, proposed by Jan et al. $(2012,2014)$ was adopted for this project. This approximation method uses the edges of a constrained Delaunay Triangulation to define the graph. A Delaunay Triangulation is defined as a triangulation in which no vertex is within the circumcircle of any triangle of the triangulation, and a constrained Delaunay Triangulation is given the obstacle edges as a constraint such that no triangulation edges cross the obstacles. By triangulating the obstacle vertices along with the origin and destination positions it is possible to create a graph representing the traversable area. In order to improve the performance of the graph and better approach the full visibility graph solution, this method includes the Fermat points of the triangles and connects these to the graph. A Fermat point is defined 


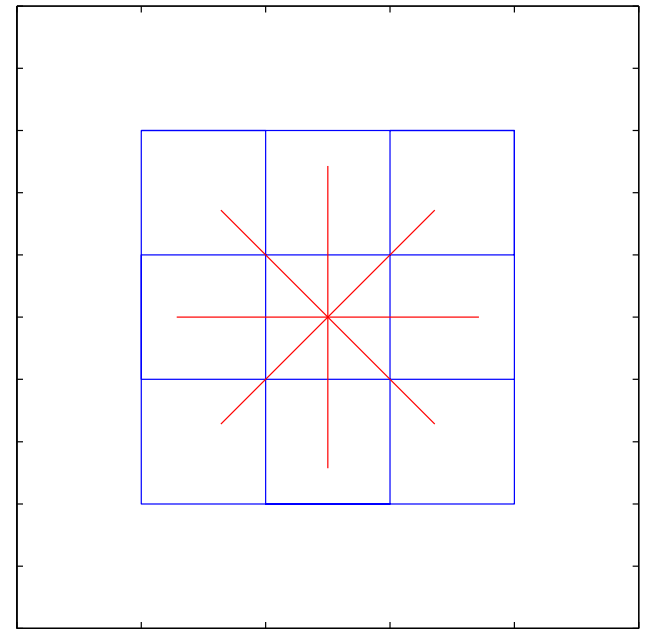

Figure 2. Grid based system allows a path to go only to one of the 8 adjacent squares surrounding it.

for triangles for which the largest angle is less than $120^{\circ}$ to be the position internal to the triangle that minimizes the distance to the triangle vertices. For a triangle in which the largest angle is greater than or equal to $120^{\circ}$ the Fermat point is located at one of the vertices. Once these Fermat points are found, they are then added to the graph and connected to their respective triangle vertices and any adjacent Fermat points (fig. 3(d) and fig. 3(e)).

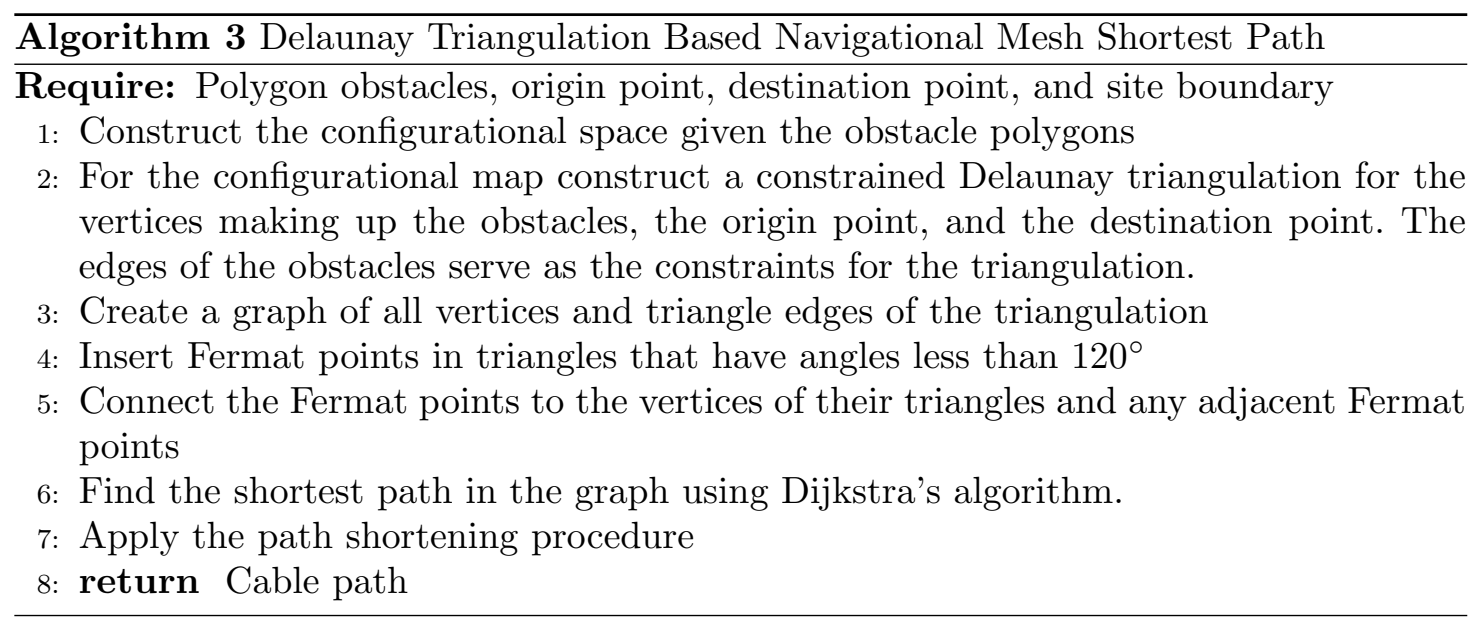

As this produces a potentially sub-optimal path, Jan et al. (2014) proposed a path shortening method which removes redundant Fermat points or vertices from the solution paths therefore reducing the total length to on average within $2 \%$ of the optimal path, but in a fraction of the time. The original path shortening algorithm was enhanced by checking all possible short-cuts, constructing a graph, and then running Dijkstra's shortest path algorithm.

Figure 3 shows a visual representation of the pathfinding process. Comparing the resulting paths in figures 3(e) and 3(f) shows the need for including the path shortening subroutine. It is important to note that inclusion of the path-shortening algorithm with the improvement suggested still does not ensure optimality, however, it can lead to significantly reduced path lengths. It should be noted that generally, however, this method 


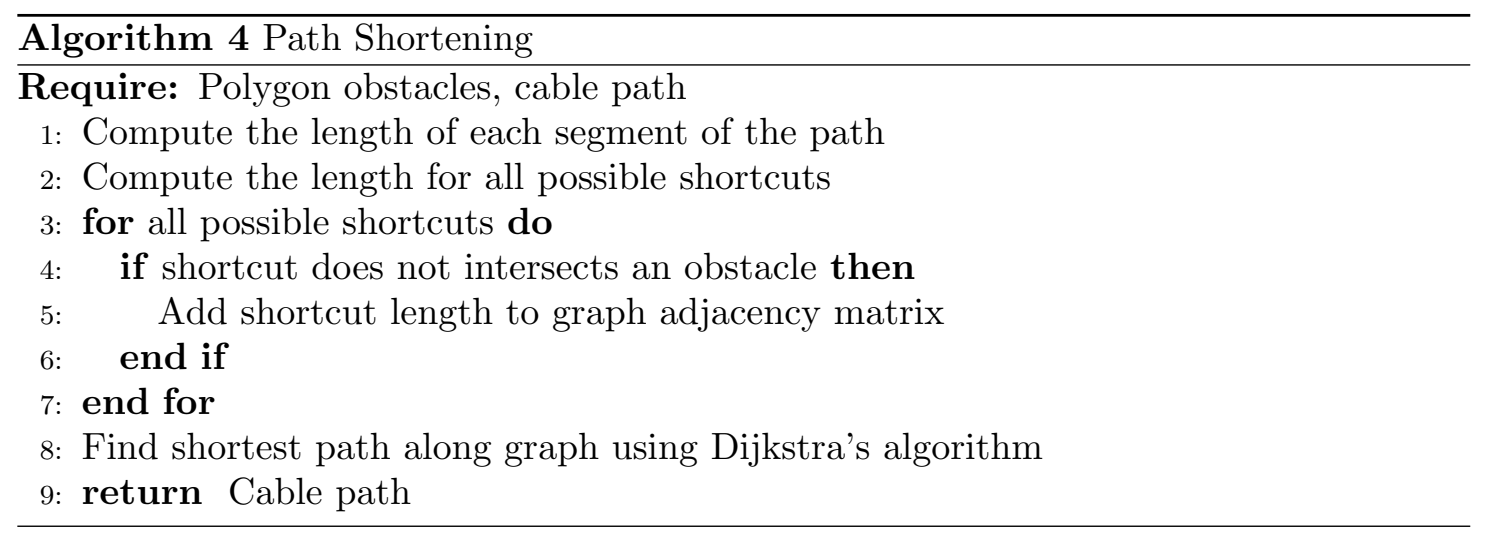

does find the optimal path between two points.

\section{MILP Formulation of Offshore Wind Farm Electrical Layout Optimization}

\subsection{Problem Description}

Through the preceding sub-problems the substations have been placed and a graph of possible cable connections has been constructed with the path and length of each cable computed. The remaining task is to select which of these cables to use to minimize the total cost of the inter-array cable infrastructure. Given the arc costs between turbines and the constraints described below, this problem could be described as a capacitated minimum spanning tree (CMST) problem with additional constraints. The minimum spanning tree problem (MST) seeks to find the sub-graph of a connected graph which connects all vertices at minimum total cost (fig. 4). The CMST variation on this problem introduces additional constraints to account for maximum capacities on the arcs. The CMST is an NP-complete problem and exact methods are often avoided though easily formulated. Similar to previous studies, the CMST was here implemented as an MILP problem and solved using the Gurobi package through MATLAB.

The CMST is not a new problem and the formulation used in this work is based on that of Gouveia $(1993,1995)$. This work has generalized this formulation to allow for multiple arc types and a simultaneous selection of not only the cable paths, but the cable cross-sectional area. 


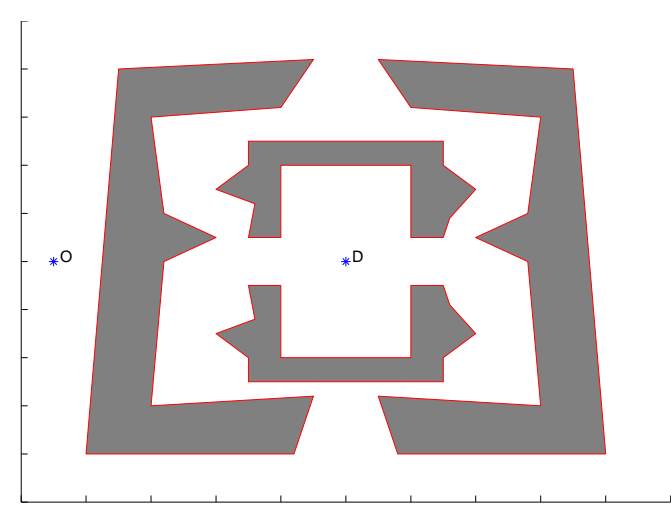

(a)

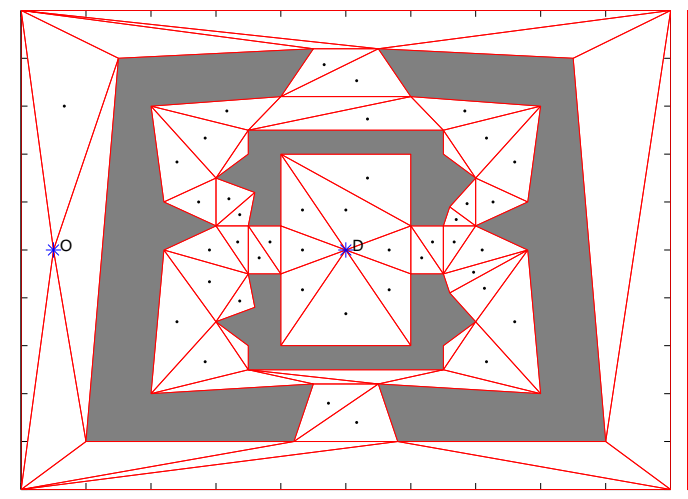

(c)

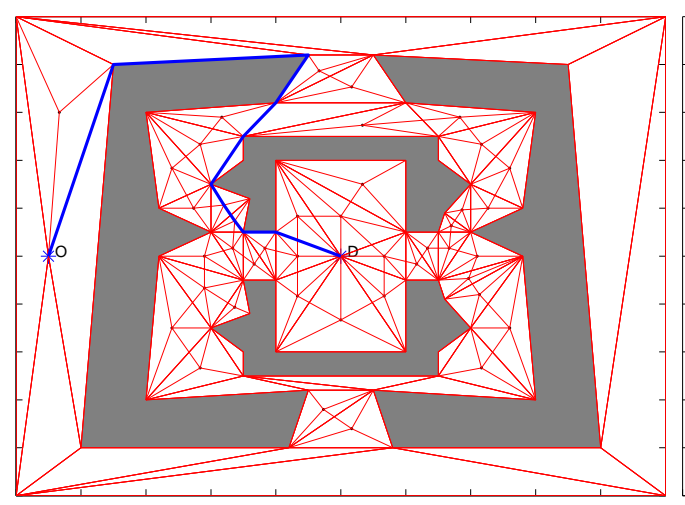

(e)

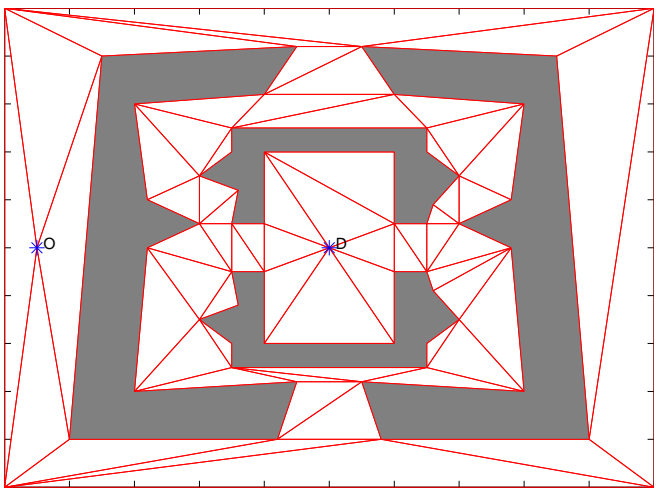

(b)

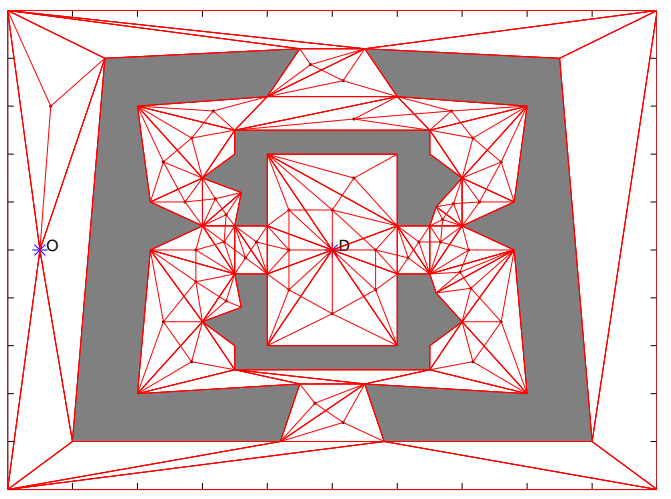

(d)

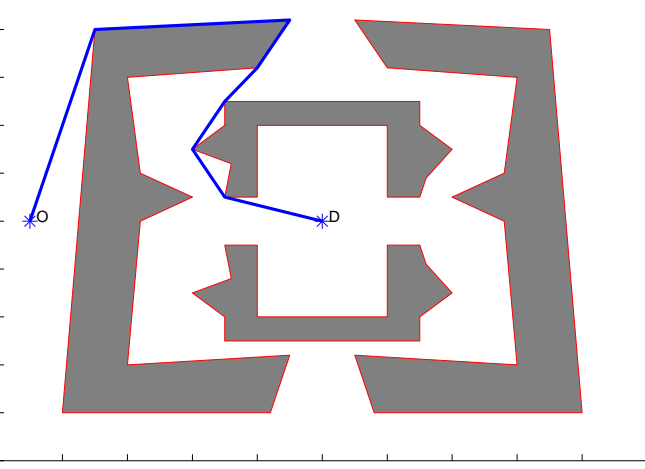

(f)

Figure 3. A simplified example of the pathfinding approach. Figure 3(a) shows the problem formulation with the origin and destination points marked and obstacles shown in grey. Figure 3(b) shows the result after performing a Delaunay triangulation on the configurational space. Figure 3(c) shows the Delaunay Triangulation with the Fermat points added for the appropriate triangles. Figure $3(\mathrm{~d})$ shows the graph formed by the triangle edges and Fermat points connected to the appropriate triangle vertices and adjacent Fermat points. Figure 3(e) shows the results from a Dijkstra's shortest path algorithm on the constructed graph and figure 3(f) shows the results after performing the path shortening function. 


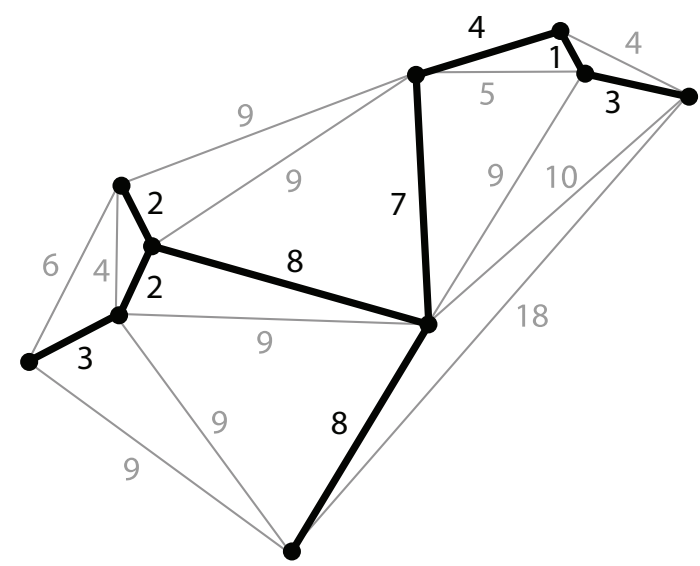

Figure 4. Example of a minimum spanning tree with arc costs shown.

\subsection{Problem Formulation}

Mathematically, the CMST can be formulated as:

$$
\begin{aligned}
& \operatorname{minimize} \sum_{i \in V} \sum_{j \in N} \sum_{l \in L}\left[\left(c_{l} \cdot d_{i, j} \cdot y_{i, j, l}\right)+\left(f_{i, j} \cdot y_{i, j, l} \cdot d_{i, j} \cdot \frac{R}{A_{l}} \cdot c_{f} \cdot I^{2}\right)\right] \\
& \text { subject to } \sum_{i \in V} \sum_{l \in L} y_{j, i, l} \leq 1 \quad \forall j \in V \text {, } \\
& \sum_{i \in V} \sum_{l \in L} f_{j, i} \cdot y_{j, i, l}-\sum_{i \in N} \sum_{l \in L} f_{i, j} \cdot y_{i, j, l}=g_{j} \quad \forall j \in V, \\
& f_{i, j}-\sum_{l \in L} Q_{l} \cdot y_{i, j, l} \leq 0 \quad \forall(i, j) \in V, \forall l \in L, \\
& \sum_{l \in L} y_{i, j, l} \leq 1 \quad \forall(i, j) \in V, \\
& \sum_{l \in L} y_{i, j, l}+y_{q, r, l} \leq 1 \quad \forall(i, j, q, r) \in X, \\
& \sum_{i \in V} \sum_{l \in L} y_{i, j, l}+y_{j, i, l} \leq Q_{\text {connection }} \quad \forall j \in T, \\
& f_{i, j} \geq 0 \quad \forall(i, j) \in V, \\
& y_{i, j, l} \in\{0,1\} \\
& \forall(i, j) \in V, \forall l \in L
\end{aligned}
$$

The above formulation represents the minimum constraints to account for a CMST with multiple arc types each with a different capacity ratings. In this formulation there are two decision variables: $f_{i, j}$ represents the the power flow between nodes $i$ and $j$ and $y_{i, j, l}$ is a binary variable representing the presence of a cable between nodes $i$ and $j$ of cable-type $l$. Both $i$ and $j$ are turbine or substation elements of the set $V$ and $l$ is a cable-type of the set $L$. The quantity $Q_{\text {connection }}$ represents the physical constraint on the number of connections at each turbine position.

The objective function is made up of two terms, the first represents the fixed capital cost of the cable and its installation where $c_{l}$ is the per-length cost of cable-type $l, d_{i, j}$ is the length of cable needed between nodes $i$ and $j$. The second term represents a factor to account for the peak losses in the cable. In this regard, the CMST is bi-objective and 
minimizes both the CAPEX costs of the cable and the losses in the cable. The losses are monetized by applying a cost of electricity $c_{f}$ to represent the forgone revenue due to the loss. The losses are computed using: $R$ is the resistivity of the cable, $A_{l}$ is the cross-sectional area of cable type $l$, and $I$ is the current level at peak, the cable length, and the flow in the cable. This bi-objective approach ensures that not only is the cable length minimized, but solutions with lower flow levels in cables are preferred in order to reduce Ohmic losses.

The seven constraints listed represent the minimum necessary for this problem including the fact that cables cannot cross one another. General CMST formulations and past wind farm planning tools do not include the constraints given by eqs. (3e) to (3g) (Gouveia 1993; Gavish 1983; Uchoa, Fukasawa, and Lysgaard 2006; Fagerfjäll 2010; Svendsen 2013). Constraint 3b stipulates that each node, or turbine can have at most one cable exporting power. Constraint $3 \mathrm{c}$ imposes the flow balance constraints such that the difference between all flow out of each node and the flow into each node must be equal to the flow supplied at each node (the power generated by the turbine) denoted by $g_{j}$. Constraint $3 \mathrm{~d}$ imposes the capacity constraint where $Q_{l}$ is the capacity of cable-type $l$. Constraint 3e ensures that every cable can be of only a single cable-type. Constraint $3 \mathrm{f}$ accounts for the fact that for an offshore wind farm inter-array cables may not cross. In order to impose this, $X$ is the set of turbine pairs for which cables cross. Constraint $3 \mathrm{~g}$ constrains the number of cables connected to a turbine to $Q_{\text {connection }}$ to account for the physical space for circuit breakers in a turbine tower. Finally eqs. (3h) and (3i) constrain $x_{i j}$ to be a positive flow, and $y_{i j l}$ to be a binary variable as explained earlier.

\subsection{Solution Approach}

Though previous work formulated the problem similarly, they identified that a heuristic algorithm would be appropriate given the NP-completeness of the problem (Svendsen 2013; Lindahl et al. 2013; Li, He, and Fu 2008). For this reason it was decided to use Gurobi 5.6, a commercial MILP solver which combines simplex solving techniques with bespoke cutting plane generation algorithms, and heuristic algorithms. Using Gurobi, the MIP gap, the relative difference between the upper and lower bounds, is used as a measure of optimality and a termination criteria. Generally Gurobi attempts to find a true global optimum which has an MIP gap approaching 0. In order to improve the performance the MIP gap was relaxed to 0.01. This means that once the upper and lower bound of the solutions are within a $1 \%$ difference the solution is considered optimal. This means in the worst case, the solution found is $1 \%$ away from optimality for the given path lengths.

Table 2. Comparison of full crossing constraint implementation to row generation method.

\begin{tabular}{rrrrrr}
\hline & \multirow{2}{*}{ Turbines } & \multicolumn{2}{c}{ Number of Crossing Constraints } & \multicolumn{2}{r}{ Time to Solve CMST $\quad[\mathrm{s}]$} \\
\cline { 3 - 6 } & & Full & Row Generation & Full & Row Generation \\
\hline & 52 & 790804 & 104 & 701.47 & 1867.68 \\
& 62 & 844914 & 2 & 847.94 & 13.79 \\
& 61 & 405862 & 0 & 1340.13 & 36.43 \\
\multirow{2}{*}{ Total } & 175 & 2041580 & 106 & 2889.54 & 1917.9 \\
\hline
\end{tabular}

As stated earlier, the crossing constraints were imposed, however, it was found during the development of the methodology that imposing the full set of crossing constraints 
for all pairs of cables resulted in many inactive constraints. It was also found that for problems with more than 40 turbines significant amounts of memory were required in order to avoid out of memory errors. It was instead decided to take an approach similar to the implementation of cutting planes and instead solve the MILP, check if any of the paths in the solution crossed, and if so impose that specific constraint. In this way the MILP solver is called iteratively, slowly increasing the number of constraints, until the solution is found. By doing this, the inactive constraints are not unnecessarily formulated and less memory is required. Even in small cases this row generation approach was shown to perform better than the full implementation. Table 2 shows a comparison of the performance using the full constraints and using the row generation approach. Due to the way in which the cable routes were found using the pathfinding algorithm described in section 4 it was not necessary to impose further constraints representing the regions where cables could not be placed.

Based on previous work by Fagerfjäll (2010) it was decided to explore the introduction of additional constraints in order to improve performance. Two additional constraints were therefore introduced:

$$
\begin{array}{ll}
f_{i, j}-\sum_{l \in L} y_{i, j, l} \geq 0 & \forall i, j \in T, \\
\sum_{i \in T} \sum_{l \in L} y_{i, j, l}+y_{j, i, l} \geq 1 & \forall j \in T
\end{array}
$$

Equation 4a relates the flow and activity of an arc, while equation 4b stipulates that there must be at least one active edge connected to each node. Neither of these constraints is necessary in order to solve the problem, however, performance improvements were noted when they were included.

\section{Results}

\subsection{Study Description}

In order to assess the performance of this approach compared to other MILP and simple estimation methodologies it was applied for a real offshore wind farm. Navitus Bay Windpark, off the south coast of England is a Round 3 wind farm site which will have between 121 and 194 turbines. The site interestingly has a number of GIS constraints that would need to be taken into account during both the siting of turbines and the design of the inter-array cable network. These GIS constraints include unexploded World War II ordnance (UXOs), ship wrecks, and areas where the seabed characteristics are unsuitable for turbines or cables.

As no decision has been made on the layout of the turbines or the size of the turbine, a realistic turbine layout was designed using WindFarmer 5.2. This layout considers only the overall site boundary and the GIS constraints and has been generated for the explicit purpose of testing this inter-array cable optimization tool; it does not represent a real layout designed by the project developer. The layout studied here consists of 175 $6 \mathrm{MW}$ turbines representing $1050 \mathrm{MW}$ installed. This layout is larger than the $968 \mathrm{MW}$ maximum allowed capacity for the wind farm and has been generated for the explicit purpose of demonstrating the capabilities of this optimization tool.

For this layout, the results using this tool are compared to running a simple design tool ignoring the GIS constraints, as well as estimating the total cable length only using the separation distance between turbines in the crosswind direction. The latter two represent methodologies often employed in layout optimization tools and cost models. The 
estimation based on the turbine separation considers neither the GIS constraints nor the capacity of cables and therefore represents a theoretical lower bound on the length of cable.

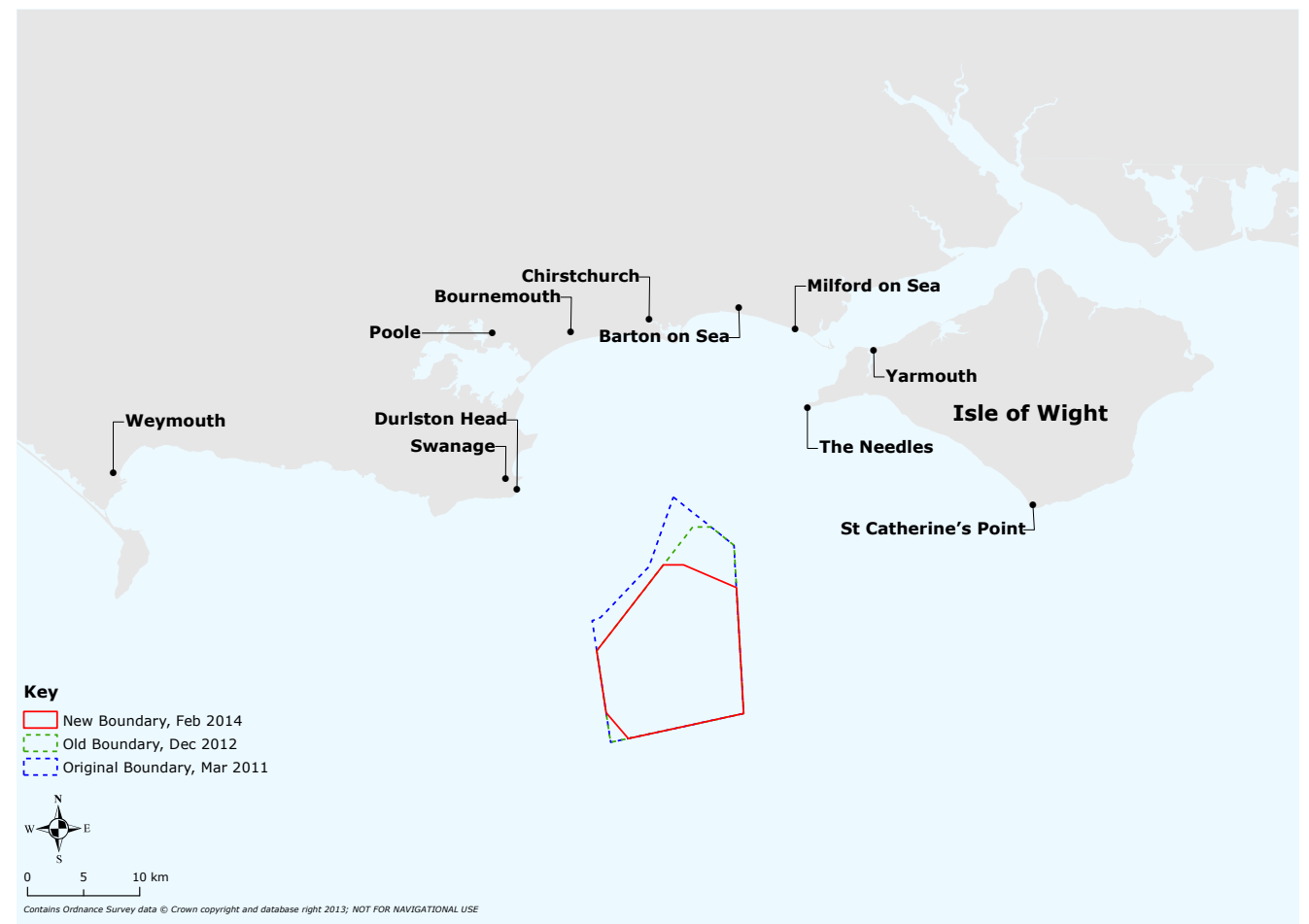

Figure 5. Illustrative map showing the Navitus Bay project site. Image courtesy Navitus Bay Development.

Based on the most recent boundaries shown in figure 5 along with the GIS data provided by the Navitus Bay Development it was possible to generate turbine layouts using DNV GL WindFarmer 5.2. These turbine positions were then input to the inter-array cable optimization tool.

All MILP optimization problems were run using a gap of 0.01. A solution is also shown using the grid based pathfinding, however, this method required the relaxation of the crossing constraint and the solutions produced by this method therefore do not represent realistic solutions.

\subsection{Substation Placement}

Running first the substation placement component of the tool allowed the new constrained capacitated kmeans $++(\mathrm{CC}-\mathrm{kmeans}++)$ algorithm to be benchmarked against common clustering approaches such as traditional kmeans and kmeans++. It should be noted that neither of these algorithms are designed to include capacity constraints or GIS based constraints limiting the area where it is permissible to place the cluster center.

Comparing the performance for a range of wind farm sizes within the Navitus Bay region it was found that the clustering was relatively inelastic to the number of turbines, and more strongly governed by the number of clusters that the turbines were to be partitioned into. Importantly, the constrained capacitated kmeans ++ approach proved to be far slower than traditional clustering approaches, however, even given this it was deemed to have an acceptable performance as 150 turbines were easily partitioned into 
two clusters in less than a second.

As can be seen in figure 6 though the performance of the new clustering algorithm is much slower than kmeans ++ , it gives similar results in terms of total distance between the turbines and the center location while at the same time adhering to the GIS and substation capacity constraints. Though the increase in computational time is relatively significant it is still a quick algorithm in absolute terms partitioning 150 turbines into two clusters in under 0.6 seconds.

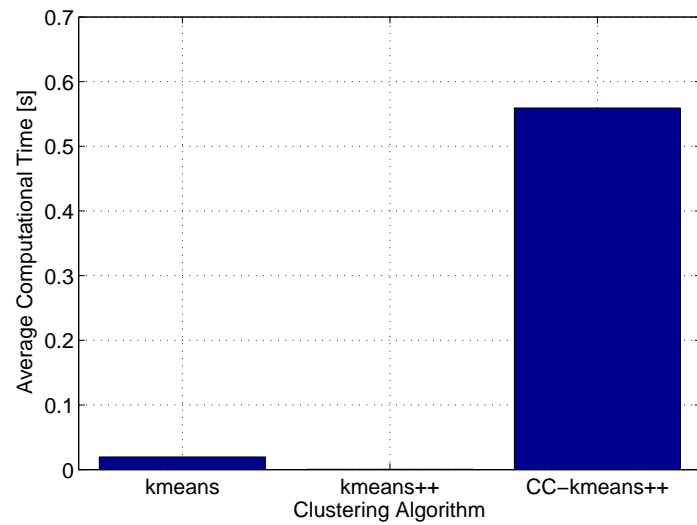

(a) Average time to partition wind farm into two clusters.

Figure 6. Comparison of the clustering algorithms. In both graphs lower values indicate better performance.

\subsection{Optimized Inter-Array Cable Layout}

The full implementation of both the substation placement and the inter-array cable optimization for a number of wind farms within the Navitus Bay site area gave the cable results shown in figures 7 and 8 . When compared to the solutions of simpler MILP programmes, ignoring GIS constraints, it was found that the total cable length increased by almost $9 \mathrm{~km}$ representing an added capital cost of approximately $€ 4.5$ million and when compared to using an estimation based on the inter-turbine spacing, the total amount of cable is increased by approximately $13 \mathrm{~km}$ representing approximately $€ 6.5$ million.

Table 3. Cable Length Comparison

\begin{tabular}{rrr}
\hline Method & Cable Length [km] & Delta $[\mathrm{km}]$ \\
\hline Turbine Spacing Based & 148.75 & - \\
CMST no GIS & 157.66 & 8.91 \\
CMST with GIS & 161.84 & 13.09 \\
\hline
\end{tabular}

From the results, a number of differences can be observed; ignoring the GIS constraints leads to a number of cables crossing the obstacle regions as would be expected. Interestingly, however, running either the $\mathrm{A}^{*}$ grid based pathfinding (fig. 9) or the navigational mesh both produce fundamentally different solutions to the cable layout problem from the base case. This can be attributed to the optimal solution being more than just rerouting the cables that violate the obstacle constraint. 


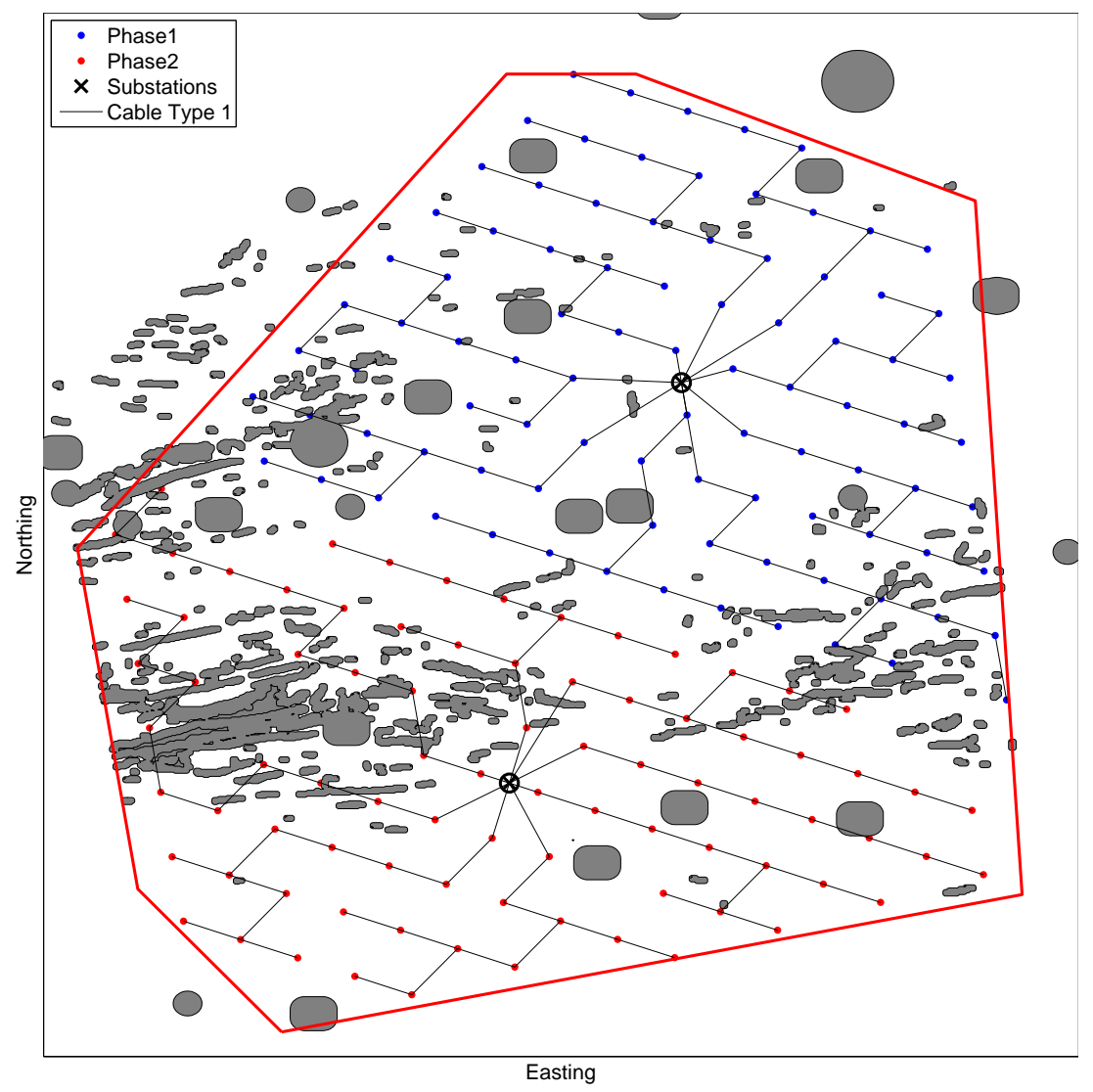

Figure 7. Cable layout, no GIS constraints.

Looking at the $\mathrm{A}^{*}$ solution shown in figure 9 , it can be observed that the grid based system experiences difficulty due to the limitations mentioned previously and in fact was unable to produce solutions without cables crossing. The proposed full methodology does, however, successfully place the substations at acceptable locations and designs an infield cable layout that does not violate any of the constraints including the GIS based constraints. This is shown in figure 8 .

\section{Conclusion}

This article has outlined a new approach for the inter-array cable design problem for an offshore wind farm by means of breaking it into several sub-problems. These sub-problems have included a location-constrained capacitated clustering approach for placing the substations, a navigational mesh based pathfinding algorithm to determine possible cable connections, and a MILP approach to solve for a CMST and select which cable connections should be installed.

The CCCP compares well in performance against traditional clustering methods such as kmeans and kmeans ++ , though consistently slower than both, it has consistently better cluster centres than kmeans, and very similar results to kmeans++ while respecting the GIS constraints. This implementation represents a novel approach to the positioning of an offshore substation and is one of the first automated approaches used for this application.

This study then opted to implement a navigational mesh pathfinding algorithm to determine possible cable connections based on constructing an approximation of a visibility graph to describe the configurational space where cables can be placed. From 




Figure 8. Cable layout, full optimization method.

the resulting graph that is constructed a simple shortest path algorithm with a bespoke path shortening heuristic is applied in order to produce good feasible solutions which approach optimality. The lengths of these paths are then used as edge lengths in an MILP implementation of a capacitated minimum spanning tree.

The results of this approach applied to a real offshore wind farm currently in the planning stages have yielded promising results indicating that this approach is not only valid but shows improvements over commonly used approaches based on the turbine separation distance. There are, still improvements that can be made, but this approach represents a strong step forward to the efficient automation of the layout design of an offshore wind farm and optimizing all aspects of the layout.

\section{Acknowledgements}

The authors would like to acknowledge EDF Energy Renewables and the Navitus Bay Project Development teams for the provision of the Navitus Bay GIS data and maps of the site.

This work is funded in part by the ETI and RCUK Energy programme for IDCORE (EP/J500847/1). 


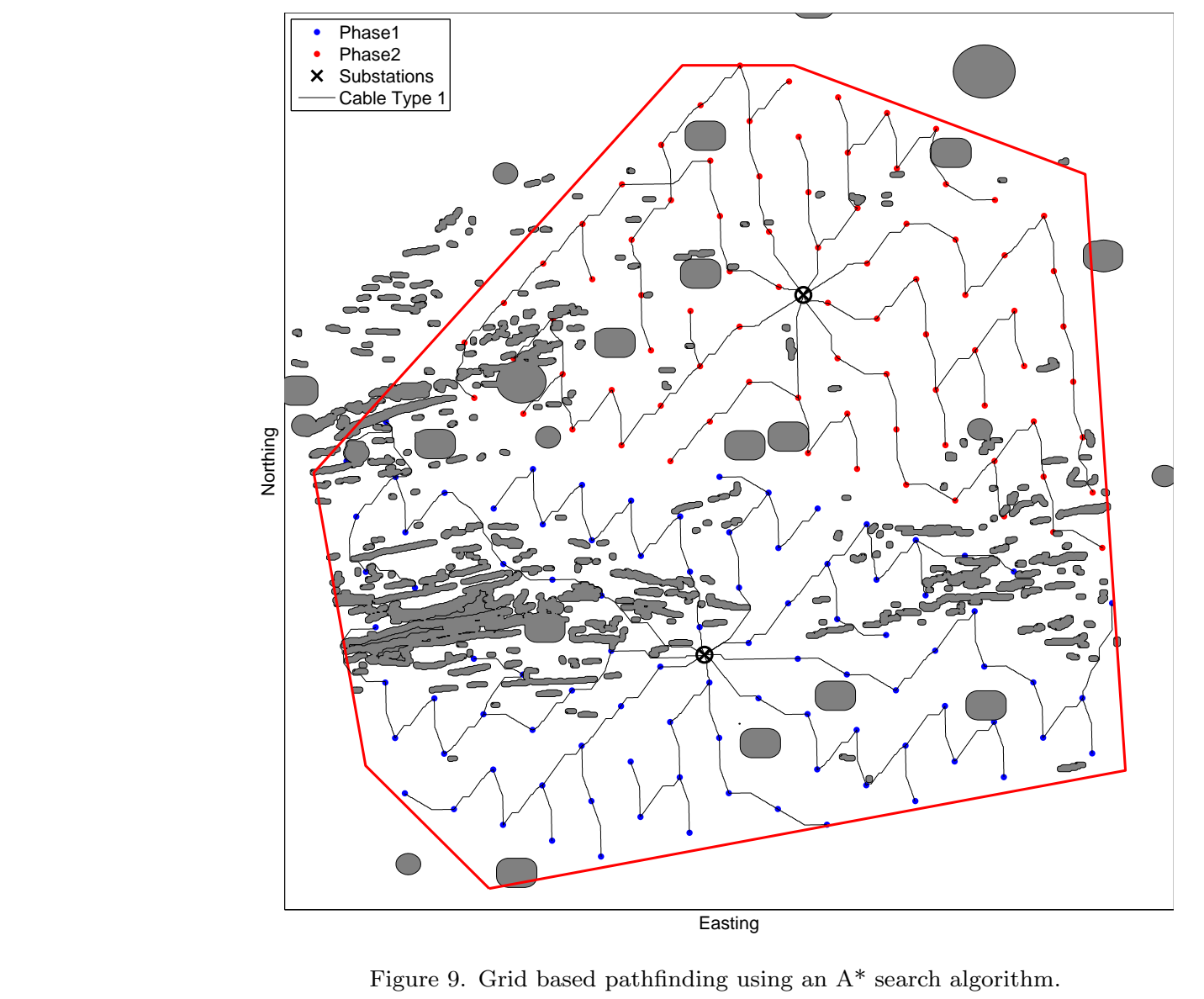

Fis.

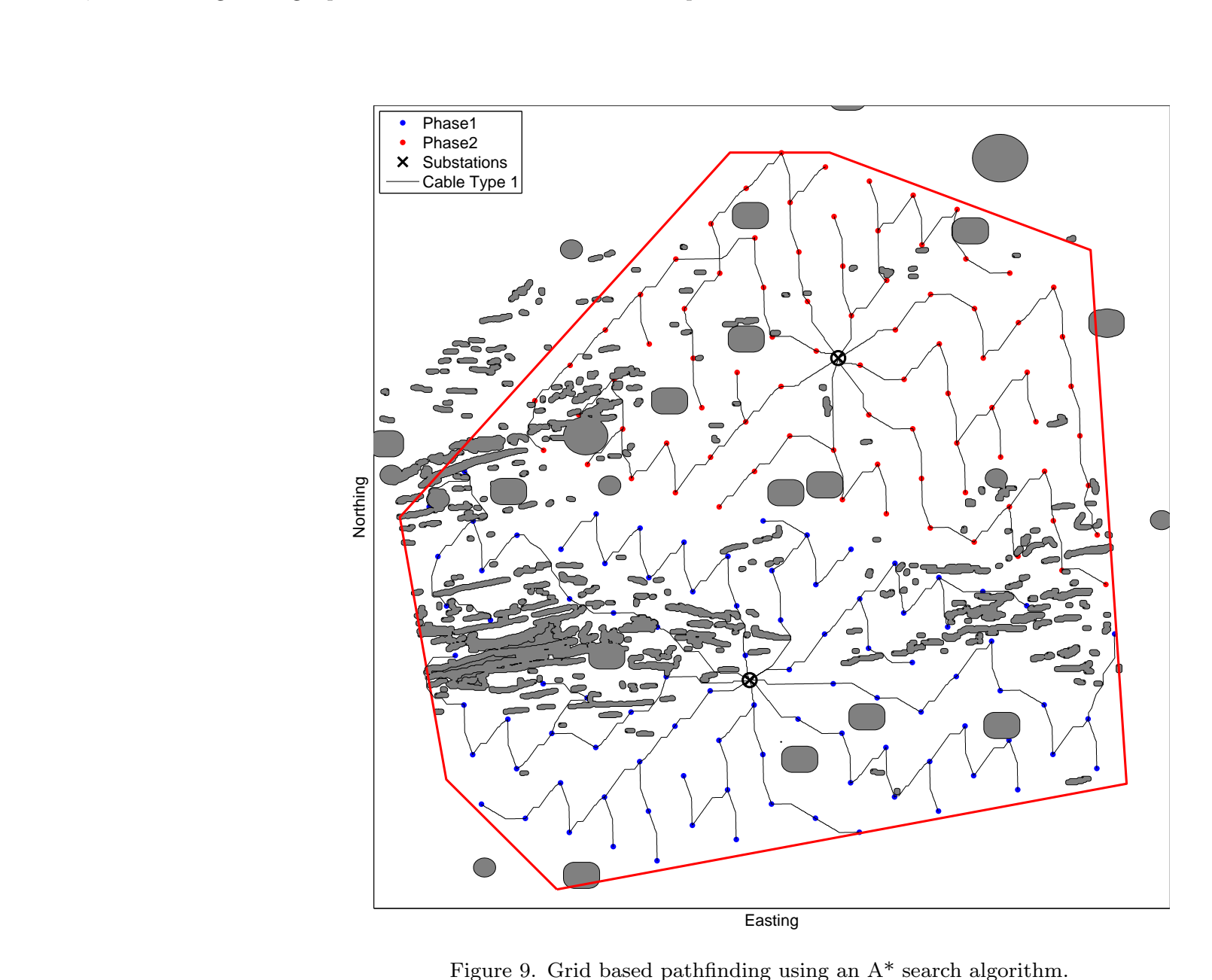

.

Tigure 9. Giid based pathfinding using an $A^{*}$ search algorithm. -

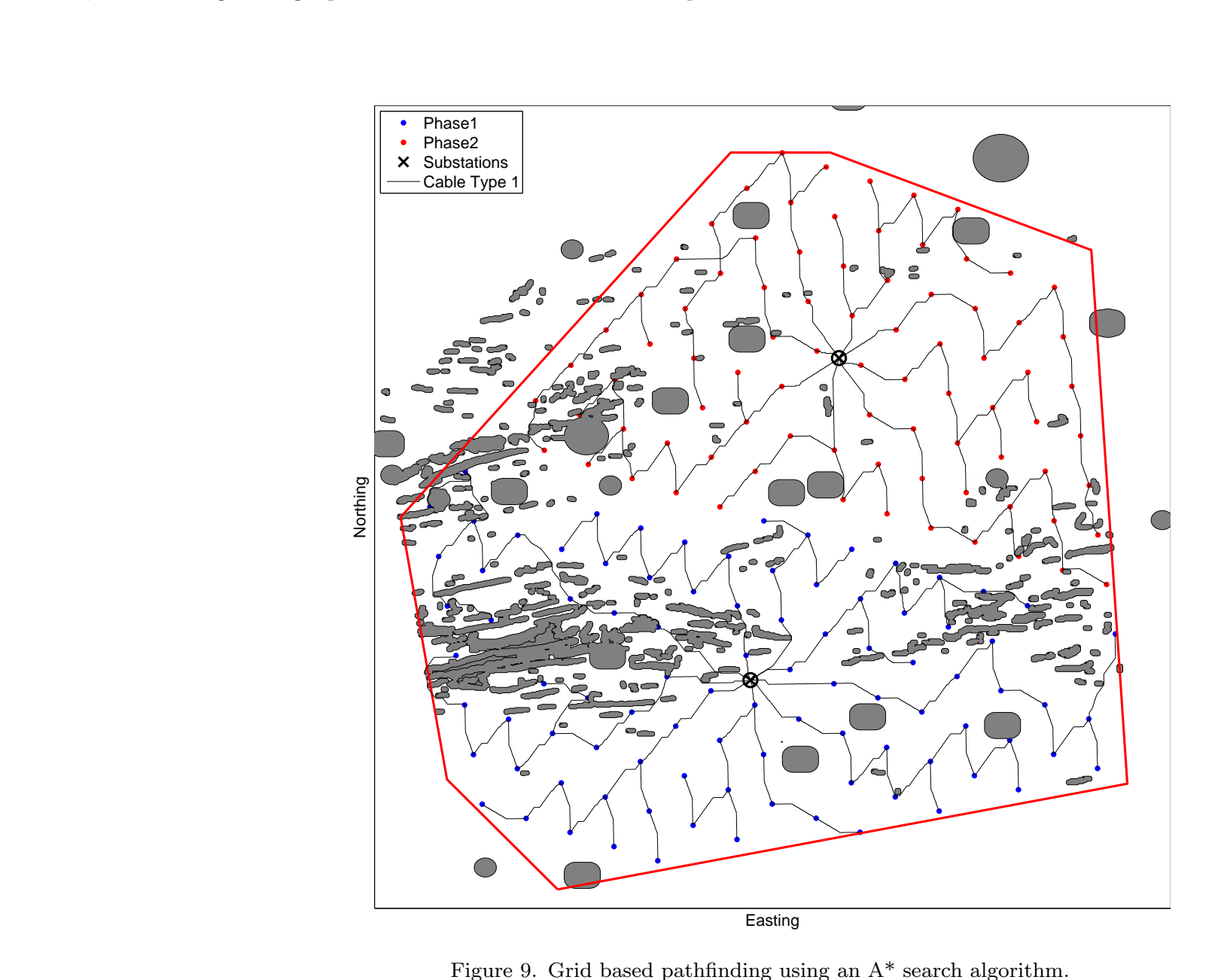

(1)

(a)

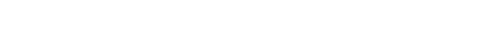




\section{References}

Arthur, David, and Sergei Vassilvitskii. 2006. k-means ++ : The Advantages of Careful Seeding. Tech. rep.. Stanford InfoLab.

Bauer, J, and J Lysgaard. 2013. "The Offshore Wind Farm Array Cable Layout ProblemA Planar Open Vehicle Routing Problem." ii.uib.no 1-16.

Burton, T, N Jenkins, D Sharpe, and E Bossanyi. 2011. Wind energy handbook. 2nd ed. John Wiley \& Sons, Ltd.

Cerveira, Adelaide, and Eduardo J Solteiro Pires. 2014. "Optimisation Design in Wind Farm Distribution Network." Proceedings of International Joing Conference SOCO'13-CISIS'13ICEUTE'13, Advances in Intelligent Systems and Computing 239.

Chamorro, Leonardo P., and Fernando Porté-Agel. 2010. "Effects of Thermal Stability and Incoming Boundary-Layer Flow Characteristics on Wind-Turbine Wakes: A Wind-Tunnel Study." Boundary-Layer Meteorology 136 (3): 515-533.

Chaves, Antonio Augusto, and Luiz Antonio Nogueira Lorena. 2010. "Clustering search algorithm for the capacitated centered clustering problem." Computers 83 Operations Research 37 (3): $552-558$.

de Berg, Mark, Otfried Cheong, Marc van Kreveld, and Marc Overmars. 2008. Computational Geometry. 3rd ed. Berlin, Heidelberg: Springer-Verlag.

Dutta, S, and TJ Overbye. 2011. "A clustering based wind farm collector system cable layout design." Power and Energy Conference at Illinois (PECI) 4-9.

Dutta, Sudipta, and Thomas Overbye. 2013. "A graph-theoretic approach for addressing trenching constraints in wind farm collector system design." 2013 IEEE Power and Energy Conference at Illinois (PECI) 48-52.

Dutta, Sudipta, and Thomas J Overbye. 2012. "Design Considering Total Trenching Length." IEEE Transactions on Sustainable Energy 3 (3): 339-348.

Elkinton, Christopher Neil. 2007. "Offshore Wind Farm Layout Optimization." Doctor of philosophy. University of Massachussetts Amherst.

Fagerfjäll, Patrik. 2010. "Optimizing wind farm layout more bang for the buck using mixed integer linear programming." Master of science. Chalmers University of Technology and Gothenburgh University.

Gavish, Bezalel. 1983. "Formulations and Algorithms for the Capacitated Minimal Directed Tree Problem." Journal of the ACM 30 (1): 118-132.

Geetha, S, G Poonthalir, and PT Vanathi. 2009. "Improved K-Means Algorithm for Capacitated Clustering Problem." International INFOCOMP Journal of Computer Science .

Ghosh, SK. 2007. Visibility algorithms in the plane. 1st ed. Cambridge: Cambridge University Press.

González-Longatt, FM, and Peter Wall. 2012. "Optimal electric network design for a large offshore wind farm based on a modified genetic algorithm approach." IEEE Systems Journal 6 (1): 164172.

Gouveia, L. 1993. "A comparison of directed formulations for the capacitated minimal spanning tree problem." Telecommunication Systems 1: 51-76.

Gouveia, Luis. 1995. "A 2n Constraint Formulation for the Capacitated Minimal Spanning Tree Problem." Operations Research 43 (1): 130-141.

Jan, Gene Eu, Chi-chia Sun, Wei Chun Tsai, and Ting-hsiang Lin. 2014. "An O (n log n ) Shortest Path Algorithm Based on Delaunay Triangulation." IEEE/ASME Transactions on Mechatronics 19 (2): 660-666.

Jan, Gene Eu, Wei Chun Tsai, Chi-Chia Sun, and Bor-Shing Lin. 2012. "A Delaunay triangulation-based shortest path algorithm with $\mathrm{O}(\mathrm{n} \log \mathrm{n})$ time in the Euclidean plane." 2012 IEEE/ASME International Conference on Advanced Intelligent Mechatronics (AIM) 186-189.

Li, DD, Chao He, and Yang Fu. 2008. "Optimization of internal electric connection system of large offshore wind farm with hybrid genetic and immune algorithm." Conference Proceedings DRPT (April): 2476-2481.

Lindahl, M., N.C. Fink Bagger, T. Stidsen, S. Frost Ahrenfeldt, and I. Arana. 2013. "OptiArray from DONG Energy." Proceedings of Wind Integration Workshop .

Lumbreras, Sara, and Andres Ramos. 2013. "Optimal design of the electrical layout of an offshore 
wind farm applying decomposition strategies." IEEE Transactions on Power Systems 28 (2): 1434-1441.

MacQueen, J. 1967. "Some methods for classification and analysis of multivariate observations." Proceedings of the fifth Berkeley symposium on Mathematical Statistics and Probability 233 (233): 281-297.

Negreiros, Marcos, and Augusto Palhano. 2006. "The capacitated centred clustering problem." Computers $\&$ Operations Research 33 (6): 1639-1663.

Svendsen, Harald G. 2013. "Planning Tool for Clustering and Optimised Grid Connection of Offshore Wind Farms." Energy Procedia 35: 297-306.

Uchoa, Eduardo, Ricardo Fukasawa, and Jens Lysgaard. 2006. "Robust branch-cut-and-price for the capacitated minimum spanning tree problem over a large extended formulation." Mathematical Programming 1-30.

Zhao, M, Z Chen, and F Blaabjerg. 2008. "Application of genetic algorithm in electrical system optimization for offshore wind farms." ... and Power ... (April): 7-12.

Zhao, M, Z Chen, and F Blaabjerg. 2009. "Optimisation of electrical system for offshore wind farms via genetic algorithm." IET Renewable Power Generation 3 (September 2008): 205-216. 